\title{
Error Analysis of Two Round Plane Circular Sensors Arrays based Acoustic Positioning System
}

\author{
Wu Song Lin, Li Li, Liu Jianxin \\ The Faculty of Mechanical Engineering Xijing University Shaanxi Xian 710123
}

\begin{abstract}
The passive acoustic locating technology is used widely in many fields. The lower locating accuracy of long distance targets location is one of the key problems that always affect its applications. In order to locate the long distance acoustic target passively, a scheme of two round plane circular arrays is presented. Each array is equate to a Vector-Sensor, could locate the direction of the target. The error source of the system is analyzed in detail. The effects of errors on the accuracy of the location are given, and then the simulation is made.
\end{abstract}

\section{Introduction}

In the passive acoustic localization and tracking technology, the design of sensor array plays a very important role. How to design a sensor array with excellent performance, simple and reasonable structure is one of key techniques to implement reliable, accurate positioning and tracking. The traditional positioning system, such as the tetrahedral, rectangular and right cross (quincunx) array, can only be used in positioning acoustic target on a limited range. In order to meet the need of large range positioning, a technical scheme of two plane circular arrays is presented. The time delay of sensors distributed at the periphery of the round plane is used to measure the direction of acoustic target. The position parameters are detected through the intersection point of two direction determined by the round plane arrays. The simulation results show that the positioning error is less than 1 meter in the area of $5 \mathrm{~km} \times 5 \mathrm{~km}$.

\section{Positioning Principles of a Double Round Plane Array}

Two round plane sensors arrays are used practically to achieve positioning, in which together $\mathrm{N}$ acoustic transducers (8 transducers are set in Figure 1) are placed equally around each round plane. An extra sensor is set at the center of each round plane.

As shown in Figure 1, original point of reference coordinate is set at the midpoint of the connection line of two round plane center. The direction of the line is defined as $\mathrm{X}$ axis. The 8 acoustic transducers are disposed equally around each round plane. On the center

\footnotetext{
a Corresponding author: 948727934@qq.com
}

of the planes, two extra acoustic sensors are set respectively. Suppose that radius of the round plane is $r$. and the center distance of two planes is D.

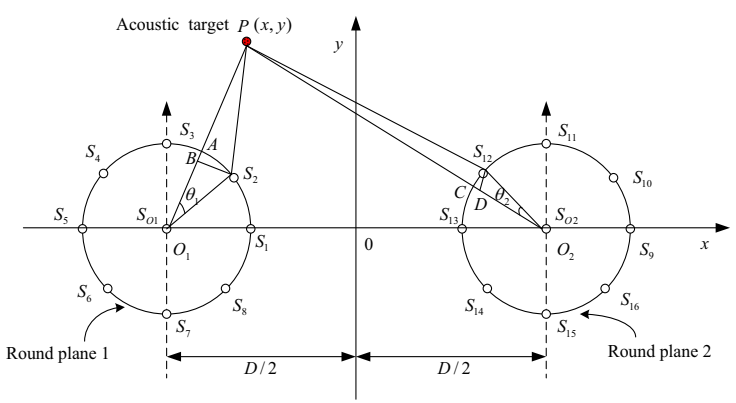

Figure 1. The disposal of acoustic transducers and sensors

The coordinates of every transducer on the plane 1 can be calculated by function (1)

$$
\begin{aligned}
x_{i} & =r \cos ((i-1) \times \theta)-D / 2 \\
y_{i} & =r \sin ((i-1) \times \theta)
\end{aligned}
$$

Similarly, the coordinates of every transducer ( ) on the plane 2 can be obtained by function (2)

$$
\begin{aligned}
x_{i+8} & =r \cos ((i-1) \times \theta)+D / 2 \\
y_{i+8} & =r \sin ((i-1) \times \theta)
\end{aligned}
$$

Where, $i=1, \cdots, 8, \theta=\frac{360}{8}=45^{\circ}$. The coordinates of transducer $S_{01}$ and $S_{02}$ are respectively $(-D / 2,0)$ and $(D / 2,0)$

An approximation condition is used when determining the azimuthal angles. The condition is that angles $\angle O_{1} B S_{2}$ 
and $\angle O_{2} D S_{12}$ were thought closely to be right angles. This approximation will bring error. The error could be very small, which is proved in the following section.

When the sound wave from source $P$ travel to the plane1 and plane2, the transducer will be triggered to record the wave coming times, $t_{1}, t_{2}, \cdots, t_{16}$ and $t_{S O 1}, t_{S O 2}$. There must be the smallest one among $t_{1}, t_{2}, \cdots, t_{8}$. Suppose $T_{\min }=t_{2}$ at a certain moment. The sound wave travel to transducer $S_{01}$. The connection line from sound source to transducer $S_{01}$ is intersected to point $\mathrm{A}$. The segment $P A$ is the shortest distance between the source $P$ and the plane 1. Point $A$ should be the nearest point to the circle of the plane 1 from $P$, the sound source. Generally, there are $P S_{2} \geq P A$, in which $P B=P S_{2}$, as the situation shown in Figure 1. Then, angle $\theta_{1}$ can be expressed as:

$$
\cos \theta_{1}=\frac{B O_{1}}{r}=\frac{P O_{1}-P B}{r}=\frac{P O_{1}-P S_{2}}{r}=\frac{C \times t_{01}-C \times t_{2}}{r}=\frac{C \times \tau}{r}
$$

Where, $\mathrm{C}$ is the sound speed in air, $\tau=t_{S O 1}-t_{2}$.

Therefore, the included angle $\varphi$, called azimuthal angle, between the straight line from the source $P$ to point $O_{1}$ and $\mathrm{x}$ axis can be expressed as (1)

When the second nearest point from the source $P$ to the plane 1 is transducer $S_{3}$, just the situation shown as Figure 1,

$$
\varphi=45^{0} \times(\text { the index of the nearest transducer }-1)+\theta
$$

If the nearest transducer is $S_{2}$ and the second one is $S_{3}$, as shown as Figure1, then

$$
\varphi=45^{0} \times(2-1)+\theta
$$

When the second nearest point from the source $P$ to the plane 1 is transducer $S_{1}$

$$
\varphi=45^{\circ} \times(\text { the index of the nearest transducer }-1)-\theta(4)
$$

Similarly, the azimuthal angle from sound source $P$ to the plane 2 can be calculated.

The slope of straight line $P 0_{1}$ from the source $P$ to $O_{1} k_{1}=\tan \varphi$ 。 Then, the function of $P 0_{1}$ is

$$
y=k_{1}(x+D / 2)
$$

Similarly, the function of the straight line $\mathrm{PO}_{2}$ from $\mathrm{P}$ to $\mathrm{O}_{2}$ can be expressed as

$$
y=k_{2}(x-D / 2)
$$

The coordinates of sound source $P$, the intersection point of two straight lines $P 0_{1}$ and $P 0_{2}$, can be obtained by solving the equations.

$$
\begin{gathered}
\left\{\begin{array}{l}
y=k_{1}(x+D / 2) \\
y=k_{2}(x-D / 2)
\end{array}\right. \\
x=\frac{D\left(k_{1}+k_{2}\right)}{2\left(k_{2}-k_{1}\right)}, y=k_{1}(x+D / 2)
\end{gathered}
$$

\section{Analysis of Positioning Error}

It could be seen that, from the expression of $\mathrm{x}$ and $\mathrm{y}$, the sources for error mainly come from the azimuthal angle $\varphi$ and time delay error $\delta_{\tau}$. In addition, the sound travel speed in air c would be affected by temperature and wind direction. And assembling precision of transducers and the round planes is another factor to impact the positioning accuracy.

The azimuthal angle $\varphi$ is determined through solving function (1) and (2). Therefore, the error of $\varphi$ mainly comes from the error of determining $\theta$, without considering the errors of time delay and the others. The angle $\theta$, however, is obtained under the approximation condition, which supposes that the angle $\angle O_{1} B S_{2}$ is a right one. So, the error produced by the condition should be determined firstly, which can be used to get the error of angle $\varphi$.

Now, a simulation method is carried out to determine the produced error. The Monte Carlo algorithm is used in digital calculation and simulation. Several known sound sources are used to compute a real angle value $\theta$. The approximated value $\theta^{\prime}$ of $\theta$ is then estimated through the approximate method. The difference between $\theta^{\prime}$ and $\theta$ is finally used to calculate the error of determining $\theta$.
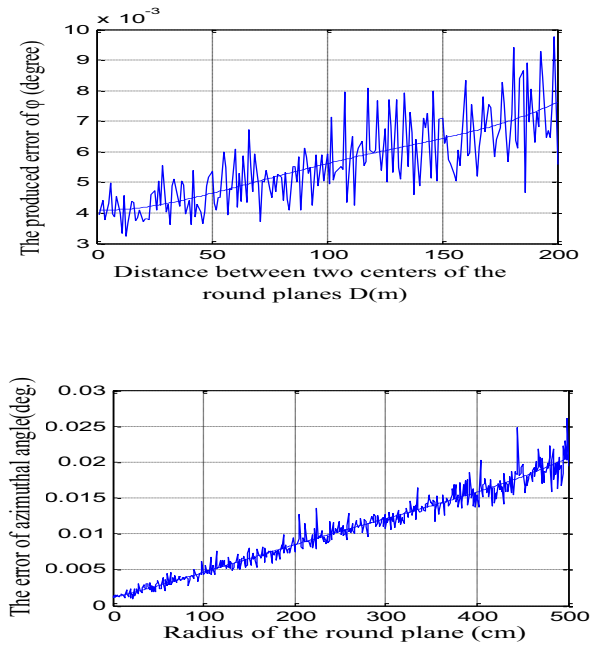

Figure 2 Estimated errors of azimuthally angle with the centers' distance $\mathrm{D}$ (up) and the radius $\mathrm{r}$ (down)

In the simulation, $\mathrm{r}=1 \mathrm{~m}, \mathrm{C}=340 \mathrm{~m} / \mathrm{s}$ and the sound source area is set as $5 \mathrm{~km} \times 5 \mathrm{~km}$. As shown in Figure 2(up), the produced error of azimuthally angle $\varphi$ increases with the increase of difference between two centers of the round planes, D. When $\mathrm{D}$, the centers distance, is the range of $0 \sim 50 \mathrm{~m}$, the amplitude of fluctuation shows relatively smooth change. It can be calculated that the average produced error of azimuthally angle $\varphi, \delta_{\varphi 1}=0.0082$ (deg. ).

As shown in Figure 2(down), the produced error of azimuthally angle $\varphi$ increases with the increase of the plane radius $\mathrm{r}$, where $\mathrm{D}=50 \mathrm{~m}, \mathrm{c}=340 \mathrm{~m} / \mathrm{s}$ and the area is still set as $5 \mathrm{~km} \times 5 \mathrm{~km}$. When $\mathrm{r}$ is in the range of $0 \sim 1 \mathrm{~m}$, the change of fluctuation shows relatively smooth. The 
average produced error of azimuthal angle $\varphi$ is $\delta_{\varphi 2}=0.0036$ (deg. ), calculated by statistical computations.

As a result, in practical application if $\mathrm{r}=0 \sim 1 \mathrm{~m}, \mathrm{D}$ $=0 \sim 50 \mathrm{~m}$, the overall error of azimuthal angle $\varphi$ is nearly $\delta_{\varphi}=\sqrt{\delta_{\varphi 1}^{2}+\delta_{\varphi 2}^{2}}=0.009$ (deg.), under the approximation condition.

The error of time delay estimated depends on applied estimation method. In reference [3], a general cross correlation method and its estimated errors are given, in which the target and noise are uncorrelated stationary random processes. When the frequency band is below $400 \mathrm{~Hz}$, sampling time interval $5 \mathrm{~ms}$, and signal and noise ratio $5 \mathrm{db}$, the error of time delay estimated could reach the lower limit, $\delta_{t 1}=3 \mu \mathrm{s}$.

In the other ways, the amplitude and phase of sound wave traveled in the air would also change randomly. The effective impedance on ground, refraction and atmospheric turbulence caused by wind and temperature gradient ${ }^{[4,5]}$ are main contribution factors. Generally, traveling time error $\delta_{t 2}$ is about $38 \sim 73 \mu \mathrm{s}$.

The error from sound measuring system, however, may come from the difference between performances of transducers and electrical components, such as the phase displacements among each transducerchannel and preamplifier circuits. The result could be compensated according to practically measuring in laboratory condition. After the compensation, the error could be controlled as $\delta_{t 3}=13-27 \mu \mathrm{s}$.

The final combined error should be controlled as $\delta_{t}=\sqrt{\delta_{t 1}^{2}+\delta_{t 2}^{2}+\delta_{t 3}^{2}}$, about $80 \mu \mathrm{s}$.

Through the foregoing analysis of various factors, positioning error comes mainly from the error of azimuthal angle and error of time delay, produced by using the approximation condition. To verify the positioning performance of the double round plane array, a Monte Carlo method is used to carry out simulation, integrated the two major factors. In the simulation, $\mathrm{D}=50 \mathrm{~m}, r=0.5 \mathrm{~m}$, error of time delay $\delta_{\tau}=1-80 \mu \mathrm{s}$, the error of azimuthal angle $\delta_{\varphi}=0.009$ (deg.)and the sound source area is set as $1 \mathrm{~km} \times 1 \mathrm{~km} \sim 10 \mathrm{~km} \times 10 \mathrm{~km}$ accordingly.

With the different sound source area, the simulation has indicated the relationship between positioning errors of coordinates $\mathrm{x}$ and $\mathrm{y}$ and sound source range, shown as Figure 3(right).
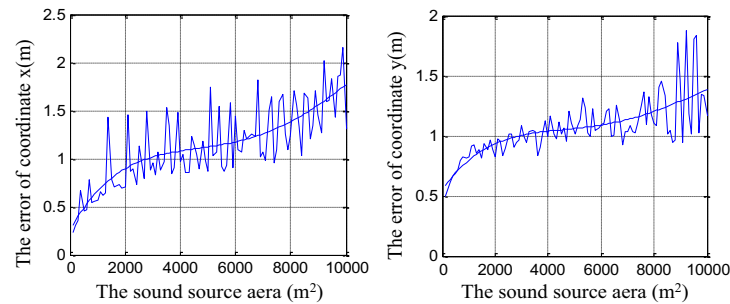

Figure 3 Changing tendency of errors of the coordinates(x,y) with sound source area

The errors of coordinates $(\mathrm{x}, \mathrm{y})$ keep increasing continuously with the increase of sound source area. When the area ranges to more than $5 \mathrm{~km} \times 5 \mathrm{~km}$, the errors of coordinates $x$ develop rapidly. In the range of $5 \mathrm{~km} \times$ $5 \mathrm{~km}$, the average errors of coordinates $\mathrm{x}$ and $\mathrm{y}$ can be resulted, $\delta_{\mathrm{x}}=0.762 \mathrm{~m} \delta_{\mathrm{y}}=0.721 \mathrm{~m}$. When the area is set as $5 \mathrm{~km} \times 5 \mathrm{~km}$, the error of time delay $\delta_{\mathrm{T}}=1-80 \mu \mathrm{s}$, the error of azimuthal angle $\delta \varphi=0.009$ (deg.), the simulation is carried out with various center distances $\mathrm{D}$ and radius $\mathrm{r}$ and the results are shown as Figure 4. It also shows that the positioning errors of coordinates $\mathrm{x}$ and $\mathrm{y}$ increase with the increase of $\mathrm{D}$ and $\mathrm{r}$.
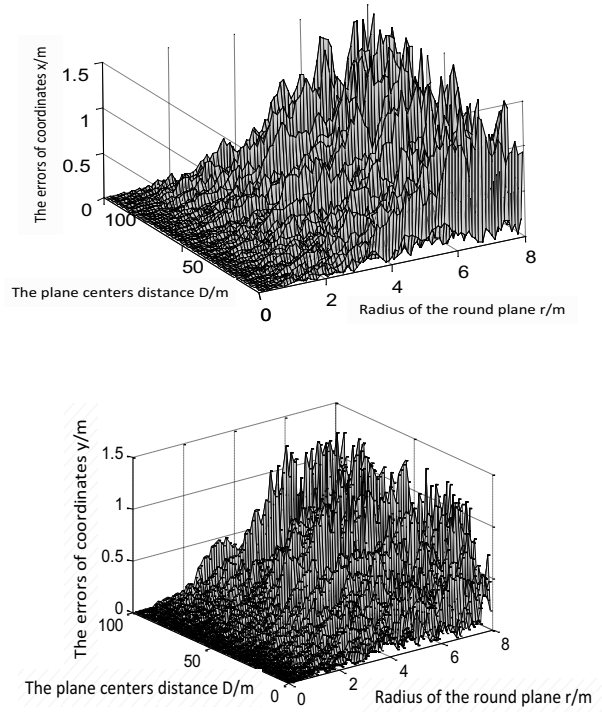

Figure 4 Series changing chart of the errors of coordinates $(\mathrm{x}, \mathrm{y})$ with $\mathrm{r}$ and $\mathrm{D}$

\section{Conclusions}

In this paper, a scheme of double round plane arrays is presented, with the properties of convenient arrangement, sample modeling and small amount of calculation to efficiently implement long distance target location. Through simulation, the performance of the method has been revealed and summarized as followings.

The positioning accuracy will reduce with the increase of planes' centers distance D and a certain value $r$, the radius of the plane. If the planes' radius $r$ and the planes' centers distance D are firmed up, the positioning accuracy will also reduce with the expanding of sound source area. In practice, the planes' radius $r$, and the centers' distance D are suited to take $0.5 \mathrm{~m}$ and $10-50 \mathrm{~m}$ respectively, in which total 8 transducers should be set along the planes' circle.

\section{References}

1. Wang S., Li H., Zhao J. The error analysis of passive acoustic positioning in $\operatorname{air}[\mathrm{J}]$.Applied Acoustics, 2000,19(2): 39-43.

2. Li Ji, Chen W.C.,Li J. Comparison Simulation of Time Delay based Passive Positioning Detecting Arrays[J].Journal of Detection\&Control, 2004, 26(1): 57-60. 
3. Charles Knapp,Glifford Carter. The generalized correlation method for estimation of time delay[J]. IEEE Trans. ASSP. 1976,24(4):320-327.

4. Daigle G A, Piercy J E, Embleton T F W. Line-ofsight propagation through atmospheric turbulence near ground[J]. J Acoust Soc Am,1983,74(5):15051513.

5. Daigle $\mathrm{G}$ A, Embleton $\mathrm{T} F \mathrm{~W}$, Piercy $\mathrm{J}$ E. Propagation of sound in the presence of gradients and turbulence near the ground $[\mathrm{J}]$. J Acoust Soc Am,1986,79(3):613-627.

6. ZHANG W P, WANG W C. The Analyze of Passive Acoustic Location in Plane by Random Shape Array of Three Sensor[J]. Journal of Detection \& Control, 2003.9, 25(3):54-57.

7. SUN S X, GU X H, SUN X X. Study on Location of Acoustic Target with a Rectangular Pyramid Array[J]. Applied Acoustics, 2006.3, 25(2): 102-107

8. Mumolo, Enzo. Algorithms for acoustic localization based on microphone array in service robotics[J]. Robotics and Autonomous Systems, 2003, 42(2): 69 88 